\title{
The Total Lymphocyte Count is a Factor When Using the CD4 Count to Guide HIV Therapy
}

\author{
Peter Hansen ${ }^{1 *}$, Dorothy Sylvia², Michelle DeLelys ${ }^{3}$ and Frederic Preffer ${ }^{4}$ \\ ${ }^{1}$ Union Biometrica Technologies, Inc., Canaan, USA \\ ${ }^{2}$ Dorothy Sylvia, PointCare Technologies, Inc., Marlborough Massachusetts, USA \\ ${ }^{3}$ Michelle DeLelys, Department of Pathology, Massachusetts General Hospital, Boston, Massachusetts, USA \\ ${ }^{4}$ Frederic Preffer, Department of Pathology, Massachusetts General Hospital, Boston, Massachusetts, USA
}

\begin{abstract}
Therapy initiation at confirmation of HIV-infection is now medically preferred, which implies that, with adequate financial support, the diagnostic use of T-helper lymphocyte parameters will shift from ART enrollment to tracking therapeutic response. Of the two main therapy tracking parameters, CD4\% and the CD4 count, the CD4\% parameter is less prone to non-HIV influences. For example, normal CD4\% values do not change at all during 17 years of healthy childhood maturation while the CD4 count falls by approximately a factor of three. In mature, HIV-stable persons the CD4 count randomly fluctuates by approximately $12 \%$ from day to day in health and by $30 \%$ in severe HIV-infection; while CD4\% fluctuates by one-third of these amounts respectively. Despite indications such as these that CD $4 \%$ is the best cell proxy for HIV-related immune status, CD4\% is not delineated in adult therapy guidelines and not universally measured by smaller commercial cytometers in resource challenged areas. With only the CD4 count available how might one determine whether a result was subject to factors that would call for a repeat count at a later time? We showed in two populations (African and American) that the normality of the total lymphocyte count was an indicator of the utility of a given CD4 count. High or low lymphocyte counts were strongly related to a high or low CD4 count, which means that factors such as stress and microbial infection that are known to temporarily affect the total lymphocyte count will affect the CD4 count. During HIV therapy the total lymphocyte count should be inclined to normal values and a lymphocyte count that is high or low relative to a patient's history would call for a repeat CD4 measurement at a later time and, more importantly, qualify the repeat measurement.
\end{abstract}

Keywords: Immune status; ART; Best cell proxy; CD4\%; CD4 count

\section{Introduction}

Studies have shown that CD4\% is an independent predictor of HIV progression and owing to its stability a useful marker for disease stage [1-3]. The maturation of a healthy child is an example of a long-term physiological change that demonstrates the superior temporal stability of CD4\%. The CD4 count for children has a reference range that is markedly age dependent; dropping progressively by a factor of 3 from newborn to 17 years. In contrast, $\mathrm{CD} 4 \%$ has a constant reference range over this same period of maturity [4,5]. The observation that CD4\% harmonizes the reference ranges for newborns, children, and adults is consistent with the hypothesis that CD4\% is a more fundamental parameter than the CD4 count in the face of progressive physiological change.

Physiological factors also cause both the CD4 count and the CD $4 \%$ to change quasi-cyclically during a 24 hour day for healthy and HIV-infected populations. In healthy controls, the CD4 count changes by approximately $60 \%$ from early morning nadir to late at night maximum, while the change in CD4\% is 6-fold less [6]. This example of superior CD4\% stability provides evidence that it is a more fundamental parameter than the CD4 count in the face of very short term physiological changes within a given day.

Because these diurnal cycles are only quasi-cyclical and are imperfectly repeated, the CD4 count and CD4\% exhibit day to day variations even when measured at the same time of day. Such day to day fluctuations in CD4 count range from approximately $12 \%$ in healthy persons to approximately $30 \%$ for severe HIV infection. In contrast, day-to-day fluctuations in $\mathrm{CD} 4 \%$ range from approximately $5 \%$ to $10 \%$ for these same persons [6,7]. These findings have been recently confirmed with modern laboratory reference systems [8]. The attenuated day-to-day fluctuation of CD4\% compared to the CD4 count provides additional evidence that $\mathrm{CD} 4 \%$ is a more stable and plausibly more fundamental immune status parameter than the CD4 count.

Recently in this journal, Achhra et al. [9] assumed CD4\% to be the best cell-based proxy for HIV disease stage and showed that, with this assumption there could be a very wide range of $\mathrm{CD} 4$ count for any given CD4\% and that CD4 count thresholds for disease management should not be used interchangeably among different ethnic populations. They found an Asian population to have lower CD4 counts than a Caucasian population for each narrow stratum of CD4\%. This study calls into question the sole reliance on CD4 counts for HIV staging and therapy management and raises interest in the utility of CD $4 \%$.

\section{Aim}

Our new study shows that if CD $4 \%$ is taken to be the best available cell-based proxy for HIV disease stage, as suggested by Achhra et al. [9], then the wide CD4 count range they observed for any given CD4\% is caused by the wide range of total lymphocyte counts that are associated with that value of CD4\%. This has implications for HIV staging in large

*Corresponding author: Peter Hansen, Union Biometrica Technologies Inc., Canaan, NY, USA 12029, Tel: 518781 3666; Fax 518781 3659; E-mail: docwph@gmail.com

Received March 15, 2013; Accepted April 17, 2013; Published April 22, 2013

Citation: Hansen P, Sylvia D, DeLelys M, Preffer F (2013) The Total Lymphocyte Count is a Factor When Using the CD4 Count to Guide HIV Therapy. J AIDS Clin Res 4: 205. doi:10.4172/2155-6113.1000205

Copyright: @ 2013 Hansen P, et al. This is an open-access article distributed unde the terms of the Creative Commons Attribution License, which permits unrestricted use, distribution, and reproduction in any medium, provided the original author and source are credited. 
populations. Adult testing and treatment guidelines for the developing world cite the CD4 count alone. Until recently, persons with no access to central laboratory facilities have had limited access to devices that measure $\mathrm{CD} 4 \%$; and when $\mathrm{CD} 4 \%$ is available it is frequently used only as ancillary information (the exception being for children). An uncritical reliance on the CD4 count without considering the total lymphocyte count is called into question by our study.

\section{Methods}

We obtained 1,454 anonymous samples from normal and immunocompromised persons in sub-Saharan Africa and analyzed them using 6 PointCare NOW ${ }^{\mathrm{si}}$ devices and 148 anonymous samples from normal and immunocompromised persons in the United States and analyzed them using a FACSCanto $\mathrm{II}^{\mathrm{mm}}$ device. Both types of devices reported the CD4 count, $\mathrm{CD} 4 \%$, and the total lymphocyte count for each sample. In the African data set, 675 samples (46\%) were from immunocompromised persons and from the United States data set, 61 samples (41\%) were from immunocompromised persons. The samples were obtained by venipuncture into vacuum-type EDTA phlebotomy tubes and maintained in liquid form. Samples were analyzed within 6 hours of phlebotomy in both the African study and United States study. The African samples were obtained from a primarily male, nominally healthy population where the main reason for immunosuppression was HIV-infection. The United States samples were obtained from approximately equal numbers of male and female persons where there were various medical factors causing immunosuppression.

\section{Analysis}

We plotted $\mathrm{CD} 4 \%$ as the $\mathrm{X}$-axis independent variable with the corresponding CD4 count as the Y-axis variable. We used color codes to group the data into three non-contiguous ranges of the total lymphocyte count. The results are shown in Figures 1 and 2. For the three ranges of total lymphocyte count, we used light blue for low $(<1,200$ lymphocytes/microliter), green for mid-normal $(1,800$ 2,200 lymphocytes/microliter) and red for high (>3,700 lymphocytes/ microliter). These total lymphocyte count ranges were chosen for

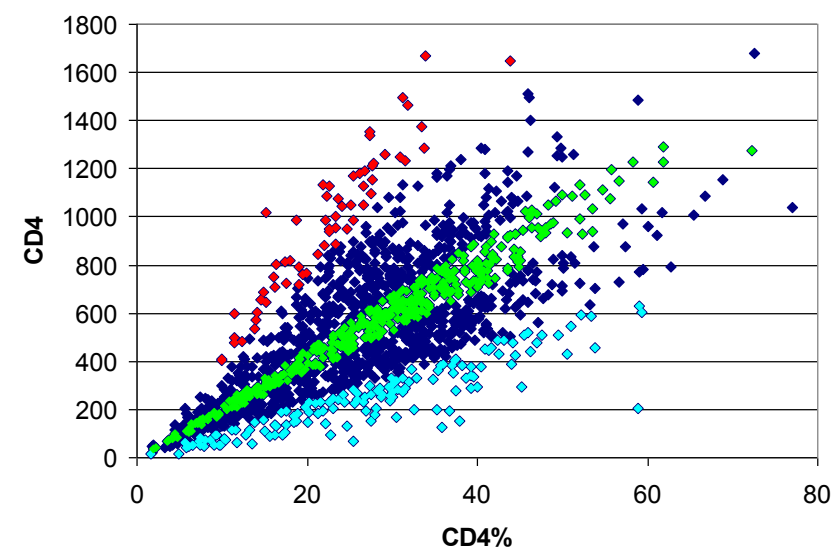

Figure 1: $C D 4 \%$ is plotted as the independent variable on the $X$-axis with the associated CD4 count plotted as the dependent variable on the Y-axis. Six PointCare NOW ${ }^{\mathrm{TM}}$ flow-cytometer/hematology devices tested 1,454 healthy and immunocompromised persons with CD4\% ranging from $5 \%$ to $75 \%$ in sub-Saharan Africa. A color code was used to identify the associated absolute lymphocyte count as low $(<1,200$ lymphocytes/microliter light blue, $10 \%$ of samples), mid-normal (1,800 - 2,200 lymphocytes/microliter green, $28 \%$ of samples) or high ( $>3,700$ lymphocytes/microliter red, $4 \%$ of samples).

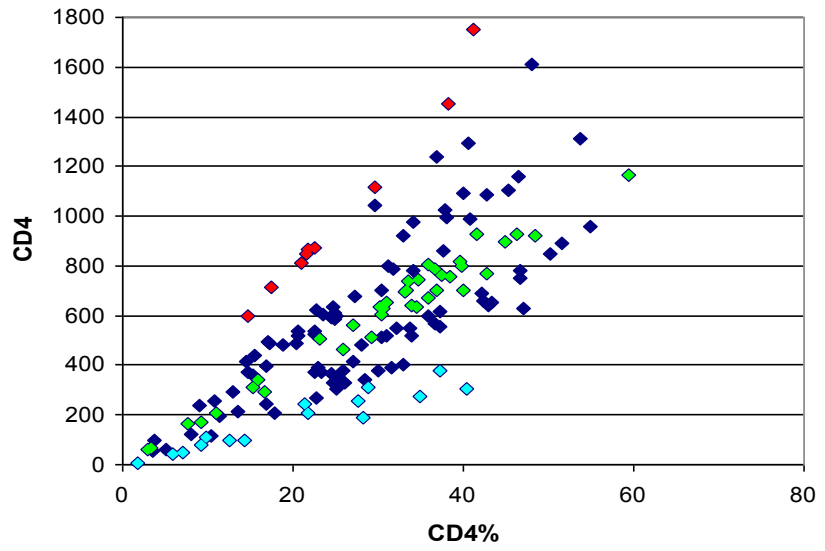

Figure 2: $C D 4 \%$ is plotted as the independent variable on the $X$-axis with the associated CD4 count plotted as the dependent variable on the Y-axis. A Becton-Dickinson FACSCanto II flow-cytometer in Boston tested 148 healthy and immunocompromised persons with CD4\% ranging from $5 \%$ to $60 \%$. A color code was used to identify the associated absolute lymphocyte count as low $(<1,200$ lymphocytes/microliter light blue, $10 \%$ of samples), mid-normal $(1,800-2,200$ lymphocytes/microliter green, $25 \%$ of samples $)$ or high $(>3,700$ lymphocytes/microliter red, $6 \%$ of samples).

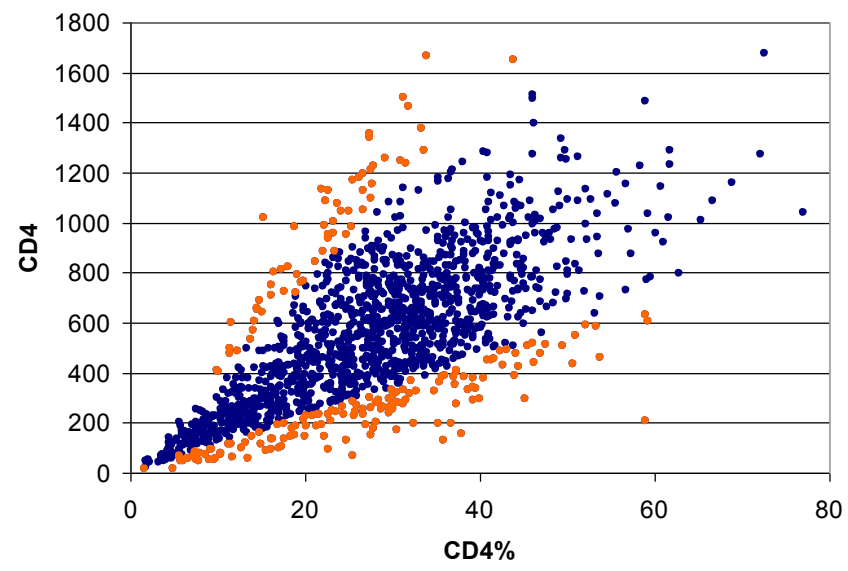

Figure 3: CD4\% is plotted as the independent variable on the $\mathrm{X}$-axis with the associated CD4 count plotted as the dependent variable on the Y-axis. Six PointCare NOW ${ }^{\mathrm{TM}}$ flow-cytometer/hematology devices tested 1,454 healthy and immunocompromised persons with CD4\% ranging from $5 \%$ to $75 \%$ in sub-Saharan Africa. The associated absolute lymphocyte counts above and below the African reference range (1,200 - 3,700 lymphocytes/microliter) are identified in orange. A total of $4 \%$ of samples were either above or below the reference range.

graphical illustration and do not correspond to sub-Saharan African or United States reference ranges.

Figures 3 and 4 are similar to Figures 1 and 2 except we indicate those samples that were above or below the reference range for the total lymphocyte count in red [10,11].

\section{Results}

Following the cited literature discussed earlier, we took CD4\% to be the stable, independent variable for immune status and observed in Figures 1-4 that for each independent value of $\mathrm{CD} 4 \%$ there is a range of observed CD4 counts which is influenced by the range of total lymphocyte counts. To illustrate our findings we constructed a CD4\% 


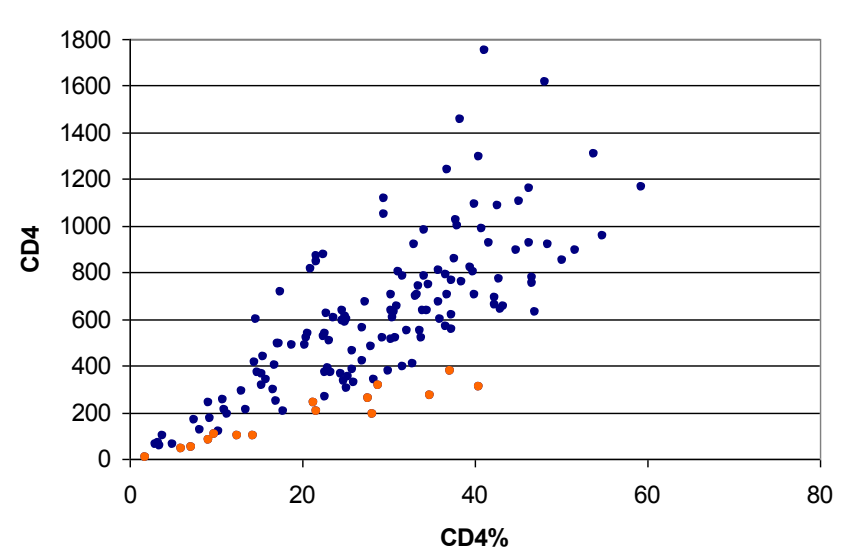

Figure 4: CD4\% is plotted as the independent variable on the $\mathrm{X}$-axis with the associated CD4 count plotted as the dependent variable on the Y-axis. A Becton-Dickinson FACSCanto II flow-cytometer in Boston tested 148 healthy and immunocompromised persons with $\mathrm{CD} 4 \%$ ranging from $5 \%$ to $60 \%$. The associated absolute lymphocyte counts above and below the American reference range $(1,200-4,800$ lymphocytes/microliter) are identified in orange. A total of $7 \%$ of samples were either above or below the reference range.

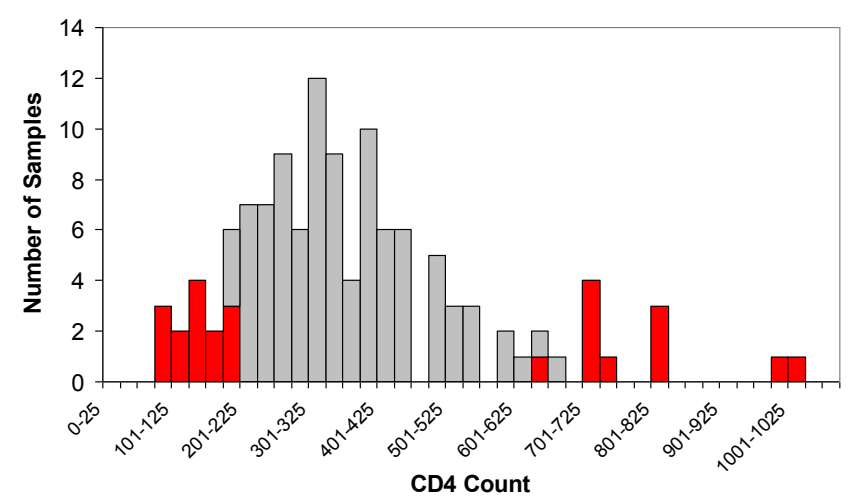

Figure 5: A histogram is shown for the distribution of CD4 counts observed when the CD4\% level was in a band between $15 \%$ and $19 \%$. The data has been drawn from the African study where the density of sample points was sufficiently high to create an illustrative histogram. The mean CD4 count is approximately 350 cells/microliter (a commonly used decision point) with approximately $2 / 3$ of CD4 counts lying between 240 cells/microliter and $470 \mathrm{cells} / \mathrm{microliter}$. CD4 counts obtained from persons with total lymphocyte counts that were outside the African reference range $(1,200-3,700$ lymphocytes/microliter) are shown with red bars.

band from $15 \%$ to $19 \%$ centered at a CD4\% level of $17 \%$. A histogram of this band is shown in Figure 5. Using only total lymphocyte counts in the normal range, we computed the mean of the associated CD4 count distribution to approximately 350 cells/microliter and the $1 \mathrm{SD}$ range to be from 244 cells/microliter to 466 cells/microliter ( $S D=111$ cells/ microliter, $\mathrm{COV}=31 \%$ ). For the United States data, the results are similar with a mean of 370 cells/microliter, a 1 SD range of 270 cells/microliter to 470 cells/microliter ( $\mathrm{SD}=100$ cells/microliter, $\mathrm{COV}=27 \%$ ). Given the fact that individuals as well as populations regularly experience total lymphocyte count variations within the normal range $[6,12]$, our study results indicate that normal range variations in the total lymphocyte count can bias CD4 counts that are near the threshold for therapy by as much as 100 counts above or below the threshold. These biases would not be apparent to a clinician that had only the patient's CD4 count available for decision making.
When we examined the effect of high and low abnormal lymphocyte counts on the range of CD4 counts for a given CD4\% the results were as follows. In the African study $4 \%$ of samples were outside the normal range for the total lymphocyte count and in the United States study 7\% were outside the normal range for the total lymphocyte count (Figures 3 and 4 ). In the African study, for a CD $4 \%$ of $17 \%$ the mean CD 4 count was approximately unchanged (370cells/microliter) from the preceding calculation but the 1SD range for CD4 count expanded to encompass the range from 186cells/microliter to 554cells/microliter, or an increase of a factor of 1.7 in SD. In the United States study the mean CD4 count was 399cells/microliter and the SD expanded by a smaller factor of 1.4 to encompass the range of approximately 261 cells/microliter to 537 cells/microliter. In certain disease conditions, which may be unrelated to HIV-infection, individuals as well as populations can experience total lymphocyte count variations that exceed the normal range. For these instances, our study results indicated that abnormal variations in the total lymphocyte count biased CD4 counts that are near the threshold for therapy by as much as 180 counts above or below the threshold. As observed above, these biases would not be apparent to a clinician that had only the patient's CD4 count available for decision making.

By taking CD4\% to be the independent variable for immune status our results reveal that in ART programs where only the CD4 count is used for therapy admission and there are no CD4\% results or guidelines available, patients with abnormal total lymphocyte counts may not be properly diagnosed. If a total lymphocyte count is available and it is high, low or abnormal based on patient history this count can be used to indicate be re-testing the CD4 count when the total lymphocyte count has returned to normal for that patient.

\section{Discussion}

Our report raises important considerations concerning the therapeutic management of HIV in worldwide, remote locations where $\mathrm{CD} 4 \%$ and the total lymphocyte count are not routinely available. Recognizing that the CD4 count is affected by many transient factors, guidelines for adult antiretroviral therapy (ART) management frequently suggest that no therapy decision be made without a repeat CD4 count measurement [13]. But how is one to know that the repeat measurement is any more reflective of a change in HIV-related immune status than was the initial measurement? Our report strongly supports the hypothesis that CD4 counts are highly influenced by total lymphocyte counts and, by extension, the variability and degree of abnormality of a repeat total lymphocyte count in a given patient can be used to determine whether the repeat CD4 count is confirmatory or not.

Quantitative studies are required to determine to what extent the number of circulating CD4 lymphocytes for an individual patient in real life has been influenced by the immune response of all lymphocytes to non-HIV related factors. With properly managed ART, HIV is a long-term disease, during the course of which competent lymphocytes continue to perform immunologic tasks; fluctuating substantially in numbers as they do so. If CD4 counts are utilized as parameters for long-term disease progression or ART monitoring these fluctuations must be better understood, or as a practical matter, coupled with $\mathrm{CD} 4 \%$ as a possible future international standard for HIV disease management. Patients residing in rural settings should have the same access to total lymphocyte counts or CD $4 \%$ as have their counterparts living near central laboratories.

The analysis and discussion in this communication strongly supports the proposal that even without computing or directly 
Citation: Hansen P, Sylvia D, DeLelys M, Preffer F (2013) The Total Lymphocyte Count is a Factor When Using the CD4 Count to Guide HIV Therapy. J AIDS Clin Res 4: 205. doi:10.4172/2155-6113.1000205

Page 4 of 4

measuring CD4\%, clinicians may more confidently answer the question as to whether they have performed sufficient confirmatory CD4 testing by consulting the repeat outcome of confirmatory total lymphocyte counts on another day. A markedly unstable or abnormal total lymphocyte count would indicate the need for more repeats until lymphocyte stability or normality is reached.

\section{Funding Support}

Partial support was provided by the Naval Health Research Center, Department of Defense Division of HIVIAIDS Prevention Program (DHAPP) under grant number NOO 244-10-1-00076; BA 10-001.

\section{References}

1. Hulgan T, Shepherd BE, Raffanti SP, Fusco JS, Beckerman R, et al. (2007) Absolute count and percentage of CD4+ lymphocytes are independent predictors of disease progression in HIV-infected persons initiating highly active antiretroviral therapy. J Infect Dis 195: 425-431.

2. Pirzada Y, Khuder S, Donabedian H (2006) Predicting AIDS-related events using CD4 percentage or CD4 absolute counts. AIDS Res Ther 3: 20.

3. Vajpayee M, Kaushik S, Sreenivas V, Wig N, Seth P (2005) CDC staging based on absolute CD4 count and CD4 percentage in an HIV-1-infected Indian population: treatment implications. Clin Exp Immunol 141: 485-490.

4. Shearer WT, Rosenblatt HM, Gelman RS, Oyomopito R, Plaeger S, et al. (2003) Lymphocyte subsets in healthy children from birth through 18 years of age: the Pediatric AIDS Clinical Trials Group P1009 study. J Allergy Clin Immunol 112: 973-980.
5. Denney TN (2005) Pediatric monitoring - CD4 issues in HIV disease. New Jersey Medical School Newark, NJ, USA

6. Malone JL, Simms TE, Gray GC, Wagner KF, Burge JR, et al. (1990) Sources of variability in repeated T-Helper lymphocyte counts from human immunodeficiency virus type 1-infected patients: Total lymphocyte count fluctuations and diurnal cycle are important. J Acq Immune Defic Syn 3: 144-151.

7. Hughes MD, Stein DS, Gundacker HM, Valentine FT, Phair JP, et al. (1994) Within-subject variation in CD4 lymphocyte count in asymptomatic human immunodeficiency virus infection: implications for patient monitoring. J Infect Dis 169: 28-36.

8. Sylvia D, Landis C, Hansen P, Branch S (2011) Improving CD4 T-cell trending in HIV patients with a moving average lymphocyte count. International AIDS Symposium, Rome, CDD070.

9. Achhra AC, Zhou J, Dabis F, Pujari S, Thiebaut R, et al. (2010) Difference in absolute CD4+ count according to CD4 percentage between Asian and Caucasian HIV-infected patients. J AIDS Clin Res 1: 1-4.

10. Karita E, Ketter N, Price MA, Kayitenkore K, Kaleebu P, et al. (2009) CLSIderived hematology and biochemistry reference intervals for healthy adults in eastern and southern Africa. PLoS One 4: e4401.

11. Wintrobe MM, Greer JP (2004) Wintrobe's Clinical Hematology. Lippincot Williams Wilkens.

12. Bull B, Korpman RA (1980) Characterization of the WBC differential count Blood Cells 6: 411-419.

13. World Health Organization (2010) Antiretroviral Therapy for HIV-Infected Adults and Adolescents: Recommendations for a public health approach. 2010 Revision. 